rockets and artificial satellites, to investigate phenomena above the Earth's surface. To get 'outside' the Earth's atmosphere to explore the external radiations space vehicles must go to heights greater than about $120 \mathrm{~km}$. Satellites cannot approach nearer than about $250 \mathrm{~km}$ if they are to remain long in orbit, and in order to get away from the ionized upper atmosphere we must use a space-probe which travels a distance of at least ten to fourteen Earth radii. With rocket-borne spectroscopes, ionization chambers and cameras, experiments have been made on observations of the ultra-violet rays and $\mathrm{X}$-rays of wave-lengths less than about $4000 \AA$ emitted from the Sun, and it has been established that the ultra-violet radiation comes only from localized areas of the Sun, and that the X-radiations of wave-lengths less than $10 \AA$ come from those active areas of the Sun which emit also radionoise of wave-length $10 \mathrm{~cm}$. As an example of what can be done with artificial satellites Dr. Ratcliffe discussed the measurement of the variation of electron concentration with height in the upper part of the Earth's atmo. sphere. The results obtained show that the electron concentration corresponds to an atmosphere the mean atomic mass of which decreases upwards, indicating that there is a gradual change from predominance in atomic oxygen at a height of about $300 \mathrm{~km}$ to predominance of helium at about $1,000 \mathrm{~km}$.

The detection of charged particles trapped in the Earth's magnetic field and the way in which these particles are trapped have been investigated by space probessatellites which travel around the Earth in very elliptical orbits but which never completely escape from the Earth's attraction. Magnetometers carried by these space probes have been used to measure the ring current, that is the sideways drift around the Earth of the positive and negative charges trapped in the Earth's magnetic field, and to investigate the magnetic field out to great distances from the Earth. It has been shown that the field decreases up to distances of from about ten to fourteen Earth radii but that beyond the decrease ceases and the field becomes irregular.

\title{
OPTICAL MASERS
}

C PHILLIPS remarks in his report (Journal of ScienJ. tific Instruments, 40, 89 ; March 1963) on the conference on optical masers held by the Institute of Physics and the Physical Society in London during September 1962, that since C. H. Townes's prediction two years ago the reaction to lasers has been exceedingly great in the United States, but comparatively only cautious in Great Britain. It is therefore not surprising, but significant, that only one of the twenty contributions to Supplement No. 1, Optical Masers, of Applied Optics*, is by a British author, but that this is the major and leading article.

The Supplement contains two long review articles and eighteen shorter papers, all dealing with various aspects of solid-state or gaseous optical masers. The first, by O. S. Heavens of the Royal Holloway College, University of London, concentrates on the basic concepts and discusses coherence, the interaction of radiation and matter, the Fabry-Perot interferometer, and oscillation conditions and methods of excitation of maser systems. The desirable spectroscopic characteristics for solid-state materials for maser application in the optical region are listed and details of the properties of crystals in use are described, together with references to the various possible applications of optical masers to physical and mathematical problems. The review, though somewhat demanding of the reader in physical and mathematical knowledge, is an excellent and comprehensive survey of the fundamental principles and of the progress of development up to September 1962. The second review, by W. R. Bennett, Yale University, is more limited in scope since he deals only with gaseous optical masers. There are four main sections. The first contains a summary of the basic considerations and the second of the dominant excitation mechanisms which have been used to produce population inversions in gas lasers. The third and main section deals with specific gaseous optical masers, in particular the helium-neon optical maser, the cæsium maser, neonoxygen and argon-oxygen masers and pure noble gas

* Optical Masers: Applied Optics. Supplement No. 1. Pp. 142. (Washington, D.C.: Optical Society of America. 1962.) 5 dollars. masers. Finally, in the fourth section, spectral characteristics and mode-pulling effects are discussed.

Solid optical masers require crystal rods with ends polished very flat and accurately parallel, and with the ends perpendicular to prism or eylinder axes. The special techniques involved and the methods used in the fabrication of such rods are briefly but clearly described in the article by W. L. Bond. Two articles deal with specific applications of ruby optical masers-the one to high-speed photography by T. Yajima, F. Shimizu and K. Shimoda, and the other as a source for the excitation of Raman spectra, by S. P. S. Porto and W. L. Wood.

In their original discussion of masers in $1958 \mathrm{M}$. Schawlow and C. H. Townes suggested the use of a FabryPerot interferometer as a multi-mode cavity and such an arrangement has since been utilized extensively. The general properties of this device when the emitting or absorbing atoms are put between the reflecting mirrors are described by A. Kastler. His paper is in French. $\mathrm{He}$ calculates the distribution of light intensity inside the interferometer and shows that the local light intensity in the stationary waves inside the cavity can be much higher than the intensity of the incident light beam. The light emitted by the atoms and emerging from the interferometer is investigated and it is shown that narrow fringes of very strong intensity can be obtained. It is emphasized that these properties are not only of application to lasers, but are also of importance in metrology and in spectroscopy.

Threshold conditions of masers for higher frequencies are discussed by K. Shimoda; population inversion in $a$ discharge in a mixture of two gases by N. G. Basov and O. M. Krokhin (this paper is printed in English and Russian in parallel columns); the coherence time of a maser, by H. E. J. Neugebauer; and non-linear optical effects, by A. E. Siegman. Scattered throughout the Supplement there is a large number of most interesting photographs of experimental optical maser set-ups, in addition to numerous references to papers on or relating to optical masers.

S. WEINTROUB

\section{CONTROL OF HYDATID DISEASE}

TTTLE is heard in most countries about movements Lo prevent the tapoworm of dogs, Echinococcus granulosus, from causing hydatid disease in human beings. The importance of this disease varies geographically in a curious manner. In most countries it is almost ignored, even when the danger is real, but in some countries it is given some attention, and in Australasia it is given a lot of attention. In New Zealand there is a National Hydatids Council, which recently published its second annual report*. * National Hydatids Council. Second Annual Report and Statement of
Accounts for the Year ended March 31, 1962. Pp. 32. (Wellington, N.Z.: Accounts for the Year end 